\title{
An Interaction Less Duo Control Strategy for Bi-polar Voltage Source Converter in Renewables Integrated Multi-Terminal HVDC (MTDC) Grids
}

This paper was downloaded from TechRxiv (https://www.techrxiv.org).

\section{LICENSE}

CC BY 4.0

SUBMISSION DATE / POSTED DATE

$30-12-2021 / 05-01-2022$

\section{CITATION}

Ancha, Satish Kumar (2022): An Interaction Less Duo Control Strategy for Bi-polar Voltage Source Converter in Renewables Integrated Multi-Terminal HVDC (MTDC) Grids. TechRxiv. Preprint.

https://doi.org/10.36227/techrxiv.17704700.v1

$\mathrm{DOI}$

10.36227/techrxiv.17704700.v1 


\title{
An Interaction Less Duo Control Strategy for Bi-polar Voltage Source Converter in Renewables Integrated Multi-Terminal HVDC (MTDC) Grids
}

\author{
Ancha Satish Kumar, Member, IEEE, and Bibhu Prasad Padhy, Member, IEEE
}

\begin{abstract}
The $P V F$ or $P V^{2} F$ double droop control is commended for its ability to regulate both the dc voltage and frequency in a decentralized approach. However, a convincing response is not achieved due to an interaction between the droop characteristics of dc voltage and frequency. This interaction affects the dc voltage and frequency support of the $\mathrm{AC}$ system surrounded Multi-Terminal HVDC (AC-MTDC) grid. To overcome this effect, a Duo control strategy is proposed in this paper, which takes advantage of a Bi-polar Voltage Source Converter (BVSC) topology in the MTDC grid. The virtue of proposed control technique is emphasized by comparing it with the existing $P V^{2} F$ double droop control along with three case studies and two test systems. The validation of interaction less Duo control strategy is carried out on five terminal CIGRE DC grid benchmark model integrated into two area power system (AC-MTDC grid-1) and New England IEEE 39 bus system (AC-MTDC grid-2). These test systems are simulated in PSCAD/EMTDC software.
\end{abstract}

Index Terms-Multi-Terminal HVDC (MTDC) grids, VSCHVDC system, dc voltage and frequency regulation, droop control, Bi-polar topology.

\section{INTRODUCTION}

$\mathbf{O}$ VER a couple of decades, the Voltage Source Converter based High Voltage DC (VSC-HVDC) systems are evolved as a proficient technology for an integration of bulk renewable sources to feed the major load centers. In contrast to the mature Line Commutated Converter (LCC) based High Voltage DC (LCC-HVDC) system, the VSCHVDC is more prominent in many aspects like power reversal without interruption, fewer footprints, ability to provide ancillary services, and black start, etc. Further, the MTDC grids improve the reliability, power trading, reduces the renewable power curtailment and variation, etc [1]. Owing to these benefits, the MTDC system is used for transmitting power from a multiple renewable sources to significant load centers [2].

To enhance the stability and reliability of a power system, extracting support from the MTDC grid is crucial during contingencies or any imbalances in a system. The MTDC grid require to fulfill the grid code requirements in order to support both the dc and ac grids [3]. Further, upon request from the Transmission System Operator (TSO), the ancillary services like frequency support, active and reactive power support, etc, need to accomplish.

Ancha Satish kumar, and Bibhu Prasad Padhy are with the Department of Electrical Engineering, Indian Institute of Technology Ropar, Punjab, India, 140001, e-mail: satish.16eez0012@iitrpr.ac.in
In the literature, researchers have achieved the ancillary service like frequency, and dc voltage support to the ac and dc grids with diverse control methods. In [4], the voltage droop, frequency droop has been combined to give frequency support to the ac grid. Change of control modes are proposed depending upon the system requirement to improve control parameters like dc voltage and frequency etc., [5]. However, the switching in control modes will not be smooth, and causes a huge transient disturbances. The alternative frequency support is provided to onshore grid while operating in active power and frequency (PF) droop instead of dc voltage and frequency $\left(V_{d c} F\right)$ droop control [6]. A data-driven analytic hierarchy process is utilized to adjust the $V_{d c} F$ droop coefficients and then optimal control to reduce the actual power deviation between demand and forecasted wind power [7]. A generalized droop control strategy proposed for improved dc voltage and powersharing in the MTDC grids [8]. A decentralized adaptive droop control strategy is proposed for enhanced dc voltage support in the MTDC grid [9]. In analogous to the frequency reserve, an over-and under-voltage reserve droop-based primary control is utilized to secure and enhance dc voltage regulation in the MTDC grid [10]. For the hybrid MTDC grids, frequency support is provided to the mainland through a comprehensive frequncy support algorithm [11]. An adaptive droop control strategy is used to improve the frequency and power-sharing in the AC-MTDC grid [12]. To enhance the frequency and dc voltage response in AC-MTDC grid, automatic generation control depending upon the model predictive control [13] and pilot voltage based droop control [14] has been used. Diverse droop controls based on communication-free techniques is discussed in [15] for GS-VSCs in the AC-MTDC grid. In [16]-[18] alike communication-free approach exploited with aid of droop control to provide inertial and frequency support to the onshore grid. A coordinated control strategy of Offshore Wind Farms (OWFs) are proposed to augment onshore grid inertial and frequency support in the VSC-HVDC system [19]. A hybrid control strategy is utilized to improve the frequency regulation of the ac system by reducing the frequency nadir in the AC-MTDC grid [20]. A supplementary control technique has been opted for emulation of inertia from Modular Multilevel Converter (MMC) and to improve frequency regulation in ACMTDC grid [21]. Autonomous frequency control is proposed for the onshore grid support through offshore HVDC grid, which works in parallel with centralized frequency control [22]. Despite the various control techniques in the literature, the $P V F$ or $P V^{2} F$ double droop control approach has its 
importance in regulating the dc voltage and frequency of $\mathrm{AC}$ surrounded MTDC (AC-MTDC) grids. However, the $P V F$ or $P V^{2} F$ droop method suffer from the control interaction, which cause the deterioted dc voltage and frequency profile in the system [23], and so become a subject mater of this paper.

The major contribution of the paper is to exploit the BVSC topology for amicable response of the VSCs to revamp the autonomous power-sharing, dc voltage, and frequency in the AC-MTDC grids. Further, the contribution of the paper are briefed as follows

1) A novel Duo control strategy that operates with individually controller for B-VSCs is proposed.

2) Out of two VSCs in B-VSC topology, one is operated in the $P V^{2}$, and another VSC is operated in the $P F$ droop control. Instead of operating both the VSCs in $P V^{2} F$ or $P V F$ double droop control methodologies.

3) Analysis has been carried out to show the interaction of $P V^{2} F$ or $P V F$ double droop control cause degradation of dc voltage and frequency support.

4) The proposed Duo strategy has been utilized in the Grid Side Voltage Source Converters (GS-VSCs). However, this control strategy can also be used in the Wind Farm Voltage Source Converters (WF-VSCs).

Moreover, steady-state and dynamic analysis has been carried out to show the effectiveness of the proposed Duo control strategy in reducing the control interaction and improving the system's stability while compared with the $P V^{2} F$ double droop control.

\section{VSC-HVDC SYSTEM MODELING}

For bulk power transmission, Bi-polar topology is preferred over a mono-pole due to its high reliability and power handing capability. Even after the outage of one VSC in B-VSCs, the converter station operates at the $50 \%$ of its transmission capability. This infers improved reliability with the Bi-polar topology. The B-VSC modeling is crucial for the system adequate operation. The detail modeling of Modular Multilevel VSCs for the system like five terminal CIGRE benchmark model as shown in Fig. 1 is burdensome with the simulation time because of its huge number of switches. The integration of this kind of dc grid to the ac grid like two area power system further worsen the situation. To reduce the burden, an equivalent MMC based VSC has to be used, and it is supposed to capture all the dynamics of detail model. Hence, in this paper, the equivalent model of type-4 proposed in [24] is adopted.

\section{VSC-HVDC GRID CONTROL}

\section{A. Generalized Control of VSC-HVDC System}

In the MMC-VSC controller, there are lower and upper level controller. The Lower Level Controller (LLC) is used to balanced capacitor voltage, circulating current suppression, and modulation techniques. However, this paper deals with the upper level controller only and henceforth, is discussed in the paper. For frequency and dc voltage regulation in decentralized manner, the droop control methodologies are admired. Instead of single droop controls like Power and dc voltage droop

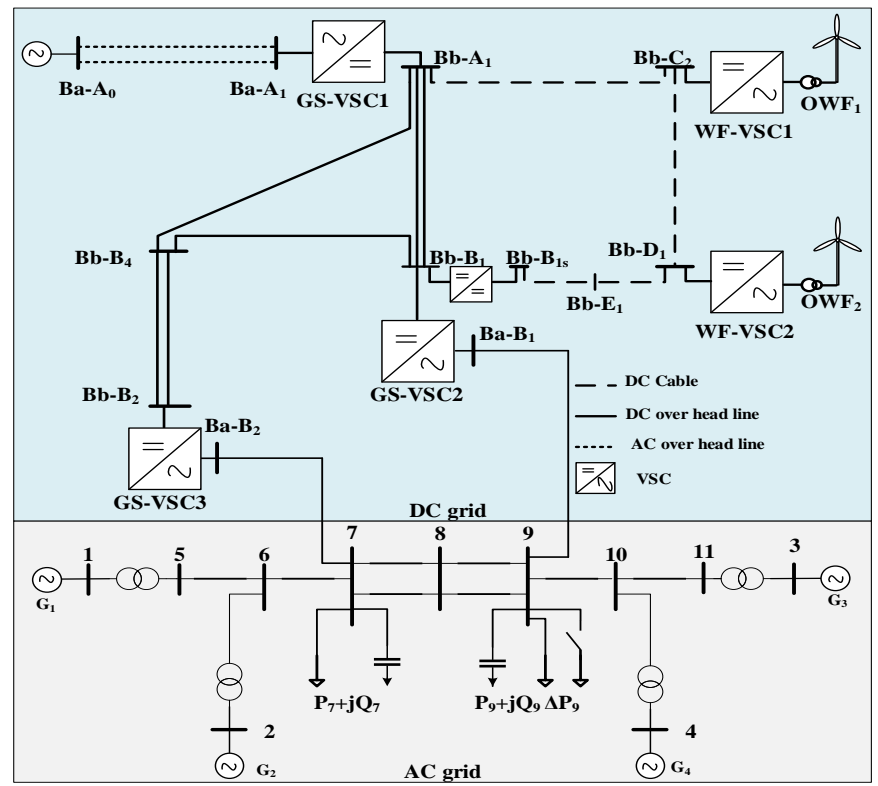

Fig. 1: AC-MTDC grid 1: Five terminal CIGRE B4 DC grid integrated into two area power system.

$(\mathrm{PV})$, power and frequency droop $(\mathrm{PF})$, and dc voltage and frequency droop $\left(V_{d c} F\right)$, the double droop controls like PVF, and $P V^{2} F$ are gaining more popularity because of its ability to support both the dc voltage and frequency in the AC-MTDC grids. The setback of these double droop control techniques is interaction among the droop control parameters, which reduce the effectiveness of dc voltage and frequency support and regulation in the AC-MTDC grid. To overcome this issue a Duo control strategy proposed in this method to enhance the support and autonomous power-sharing for the AC-MTDC grid.

\section{B. The Proposed Interaction-less Duo Control Methodology}

Any abrupt load changes or contingencies like converter outage cause the imbalances in dc grid. This ultimately results in the dc voltage and frequency deviations from their nominal values, respectively. In this situation, regulation of dc voltage and frequency along with autonomous power-sharing is requisite for the stability and reliability of the AC-MTDC grids. The proposed Duo control strategy ameliorate the dc voltage and frequency regulation in comparison with the PVF and $P V^{2} F$ double droop control, and eradicates the interaction of droop control parameters in these droop methods. The proposed Duo control strategy is as in Fig. 2. Two individual controls are used for each pole in B-VSC i.e., $P V^{2}$ and PF droop control. While operating one of the VSC with $P V^{2}$, and another VSC with $\mathrm{PF}$ droop control enhances the dc voltage and frequency support in the AC-MTDC grid. The control equations of the Duo control method are as follows

$$
\begin{gathered}
\left(\left(P^{*}-P\right)+K_{V}\left(\left(\frac{V_{d c}^{*}}{2}\right)^{2}-\left(\frac{V_{d c}}{2}\right)^{2}\right)\left(K_{p}+\frac{K_{i}}{S}\right)=i_{d 1}^{*}\right. \\
\left(\left(P^{*}-P\right)-K_{f}\left(f^{*}-f\right)\right)\left(K_{p}+\frac{K_{i}}{S}\right)=i_{d 2}^{*}
\end{gathered}
$$

Where, superscript ${ }^{*}$ ' of $\mathrm{P}, V_{d c}$, and $\mathrm{f}$ are references values of real power, dc voltage, and frequency, respectively. The $\mathrm{P}$, 


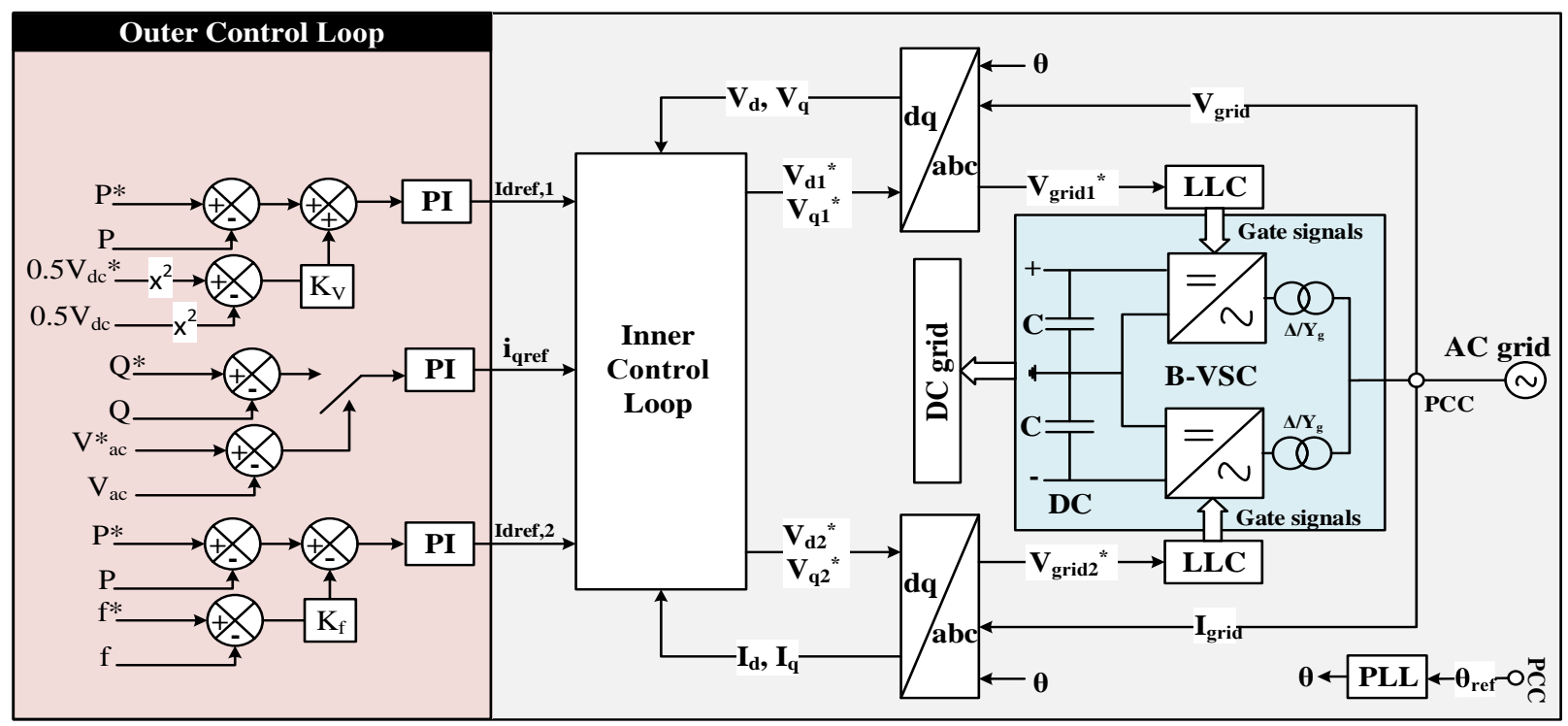

Fig. 2: The Proposed Duo Control Strategy

$V_{d c}$, and $\mathrm{f}$ are corresponding measured values. The $i_{d 1}^{*}$, and $i_{d 2}^{*} \mathrm{~d}$-axis references of each VSC in B-VSC. In this proposed control method instead of using a single control equation for B-VSCs as in equation (3), two different control equations are utilized as in equations (1), and (2).

$$
\left(\left(P^{*}-P\right)+K_{V}\left(\left(\frac{V_{d c}^{*}}{2}\right)^{2}-\left(\frac{V_{d c}}{2}\right)^{2}\right)-K_{f}\left(f^{*}-f\right)\right)\left(K_{p}+\frac{K_{i}}{S}\right)=i_{d}^{*}
$$

This approach has various advantages over the conventional double droop control method and are as follows

1) This proposed Duo control strategy eradicates the interaction due to the double droop control in the ACMTDC grid, which is responsible for deterioration of the dc voltage, frequency support, and autonomous powersharing.

2) Duo control methodology improves dc voltage and frequency support along with autonomous power-sharing in compared with other various droop control methods like $P V^{2} F$ [4], PVF [12], $V_{d c} \mathrm{~F}$ and PF [6].

3) Transmission System Operator (TSO) has more degree of freedom in choosing the control parameters as positive and negative pole in B-VSC can operate and control independently.

4) In the $P V^{2} F$ control, trade-off between the control parameters are prominent accordingly droop constants are chosen. In contrast, with the proposed control method equal importance can be provided by avoiding trade-off between dc voltage and frequency control. This way it helps TSO to avoid the trade-off issue.

5) The proposed control method does not reliant on dedicated communication system. As it operates based upon locally measured parameters.

6) Moreover, the proposed Duo control strategy is simple and proficient way to enhance the dc voltage and frequency support for the AC-MTDC grid.

Further, in most of the literature either dc voltage or frequency support is given priority but not the both parameters. However, the proposed Duo control strategy is prominent in regulating both dc voltage and frequency parameters in the AC-MTDC grids. Henceforth, the proposed Duo control method has its significance, and surpass the conventional approach of utilizing droop control for B-VSCs with an interaction-less Duo control strategy for maintaining dc-voltage and frequency in the ACMTDC grids.

\section{Analysis of Conventional $P V^{2} F$ droop Control in Compar-} ison with the Proposed Interaction-less Duo Control Strategy

1) Steady-state Analysis: In conventional double droop control approach either it is a $P V^{2} F$ or PVF, there exist an interaction among the droop control parameters, and it restrict the effectiveness of dc voltage and frequency support. To understand this, let us consider equation (3), and it can be rewritten during steady-state for B-VSCs operating in $P V^{2} F$ control mode as

$$
\Delta P_{i}=-K_{V, i} \psi_{i}\left(V_{d c}\right)+K_{f, i} \Delta f_{i}
$$

Here

$$
\psi_{i}\left(V_{d c}\right)=\left(\left(\frac{V_{d c, i}^{*}}{2}\right)^{2}-\left(\frac{V_{d c, i}}{2}\right)^{2}\right)
$$

where, $\mathrm{i}$ is the number of B-VSCs converters in the dc grid that varies from 1 to $\mathrm{n} . \Delta P_{i}$ and $\Delta f_{i}$ are deviation of power and frequency, respectively. The $K_{V, i}$ and $K_{f, i}$ are dc voltage, and frequency droop constants. These droop constants are zero for B-VSCs operating in the constant dc voltage and power control modes. Due to the presence of swing or slack converter, the sum of power deviation at the terminals of the B-VSCs in MTDC grid is zero i.e., $\sum_{i=1}^{n} \Delta P_{i}=0$. With this assumption, the equation (4) can be written as

$$
-K_{V, i} \psi_{i}\left(V_{d c}\right)+K_{f, i} \Delta f_{i}=0
$$

Now, let us consider there are ' 1 ' converters connected to the single ac area. The change in frequency is same through the ac grid in a single synchronous area i.e., $\Delta f_{i}=\Delta f$. With 
this fact, for the 'l' B-VSCs connected in single ac grid. The equation (4) can be re-written as follows

$$
\Delta f=\sum_{l=1}^{m} \frac{K_{V, l}}{K_{f, l}} \psi_{l}\left(V_{d c}\right)
$$

where, $1=1,2$..m B-VSCs in a single synchronous area. While substituting the equation (7) i.e., $\Delta f$ in equation (4) for the single ac area is given as

$$
\Delta P_{l}=-K_{V, l} \psi_{l}\left(V_{d c}\right)+K_{f, l} \sum_{l=1}^{m} \frac{K_{V, l}}{K_{f, l}} \psi_{l}\left(V_{d c}\right)
$$

The relationship between power deviation for the corresponding dc voltage deviation for the ' $\mathrm{m}$ ' $\mathrm{B}-\mathrm{VSC}$ is given by rewriting the equation (8) as

$$
\Delta P_{a c 1}=\sum_{l=1}^{m} \Delta P_{l}=\left(-K_{V, l}+K_{f, l} \sum_{l=1}^{m} \frac{K_{V, l}}{K_{f, l}}\right) \psi_{l}\left(V_{d c}\right)
$$

Further, for the simplicity $\left(-K_{V, l}+K_{f, l} \sum_{l=1}^{m} \frac{K_{V, l}}{K_{f, l}}\right)$ is replaced with $K_{v f}$ in the above equation (9) will be

$$
\Delta P_{a c 1}=K_{v f} \psi_{l}\left(V_{d c}\right)
$$

In similar manner, the relationship between the power and frequency deviation is given as

$$
\Delta P_{a c 1}=\sum_{l=1}^{m} \Delta P_{l}=\sum_{l=1}^{m}\left[K_{f, l}-\left(K_{V, l} \frac{\sum_{l=1}^{m} K_{f, l}}{\sum_{i=1}^{n} K_{V, i}}\right)\right] \Delta f_{a c 1}
$$

Again, for the simplicity $\sum_{l=1}^{m}\left[K_{f, l}-\left(K_{V, l} \frac{\sum_{l=1}^{m} K_{f, l}}{\sum_{i=1}^{n} K_{V, i}}\right)\right.$ is replaced with $K_{f v}$ in the above equation (11) that can be rewritten as

$$
\Delta P_{a c 1}=K_{f v} \Delta f_{a c 1}
$$

It is evident from $K_{v f}$, and $K_{f v}$ equivalent droop constants that the interaction among the droop control parameters reduce the equivalent dc droop, and frequency droop constants by $\left(K_{f, l} \sum_{l=1}^{m} \frac{K_{V, l}}{K_{f, l}}\right)$, and $\left(K_{V, l} \frac{\sum_{l=1}^{m} K_{f, l}}{\sum_{i=1}^{n} K_{V, i}}\right)$, respectively. This will worsen the dc voltage and frequency support to the AC-MTDC grids. However, with the proposed Duo control method, this interaction is eradicating permanently while operating individual frequency, and dc voltage droop control for each VSC in B-VSCs say positive pole is operating in the $P V^{2}$ droop control, and negative pole is operating in the $P F$ droop control. The relationship between the power, dc voltage, and frequency deviation with the proposed Duo control, the equations (1), and (2) can be re-written in steady-state as

$$
\begin{gathered}
\Delta P_{p, i}=K_{V p, i} \psi_{i}\left(V_{d c}\right) \\
\Delta P_{n, i}=K_{F n, i} \Delta f_{i}
\end{gathered}
$$

where, $\Delta P_{p, i}, \Delta P_{n, i}$ are power deviation seen by the positive, and negative pole of B-VSC. The $K_{V p, i}$, and $K_{F n, i}$ are dc voltage, and frequency droop constants used in positive, and negative pole of B-VSC, respectively.
2) Dynamic Analysis: The dynamic analysis of the proposed Duo and $P V^{2} F$ droop control strategy perceive through the simplified model of the AC-MTDC grid. This model considers the system's lower order dynamics and outer loop control of the VSCs. While modeling this AC-MTDC grid, the assumptions taken in [25] are adopted for the DC grid. Whereas, in the case of the AC system modeling, the load frequency model is utilized, and reactive power control parameters are neglected. With these assumptions, the following equation gives the modeling of a simplified AC-MTDC grid.

$$
\Delta f_{I}=\frac{K_{p, I}}{1+s T_{p, I}} \Sigma \Delta P
$$

where, $\Sigma \Delta P=\Delta P_{m, I}+\Delta P_{G S V S C, i}+\Delta P_{S C, k}-\Delta P_{L, I} \pm \Delta P_{t i e, I}$

$$
\Delta V_{d c}=\frac{p o l}{s T_{\mathrm{DC}, \mathrm{p}}+\Sigma K_{v}}\left[\left(\sum_{i=1}^{n} k_{f, i}-\sum_{i=1}^{n} k_{I F, i}\right) \Delta f_{I}\right]
$$

Whereas, in case of the Duo control strategy, the equation (16) can be re-written with the interaction factor $\left(k_{I F}\right)$ is zero as

$$
\begin{gathered}
\Delta V_{d c}=\frac{p o l}{s T_{\mathrm{DC}, \mathrm{p}}+\Sigma K_{v}} \sum_{i=1}^{n} k_{f, i} \Delta f_{I} \\
\Delta P_{m, I}=\frac{1}{1+s T_{\mathrm{tg}, \mathrm{I}}}\left[\frac{\Delta f_{I}}{R_{I}}\right] \\
\Delta P_{G S V S C, i}=-\frac{p o l}{1+s T_{\mathrm{GSVSC}, \mathrm{i}}}\left(k_{v, i} \psi\left(V_{d c}\right)+k_{f, i} \Delta f_{I}\right)
\end{gathered}
$$

This equation (19) gives the change in power at GSVSCs operating in the $P V^{2} F$ droop control. However, with the duo control strategy operating with individual converter for each pole in B-VSC, this equation can be re-written for each positive and negative pole as

$$
\begin{gathered}
\Delta P_{G S V S C, p i}=-\frac{1}{1+s T_{\mathrm{GSVSC}, \mathrm{pi}}} k_{v, i} \psi\left(V_{d c}\right) \\
\Delta P_{G S V S C, n i}=-\frac{1}{1+s T_{\mathrm{GSVSC}, \mathrm{ni}}} k_{f, i} \Delta f_{I} \\
\Delta P_{t i e, I}=\frac{2 \pi T_{I J}}{s}\left[\Delta f_{I}-\Delta f_{J}\right]
\end{gathered}
$$

where, $\mathrm{I}=1,2, \ldots \mathrm{J}, \mathrm{AC}$ areas in the $\mathrm{AC}$ grid, and $\mathrm{pol}$ is number of VSC poles (which is one for monopole and two for bi-pole VSC). $\Delta f_{I}, \Delta V_{d c}, \Delta P_{m, I}, \Delta P_{L, I}, \Delta P_{t i e, I}$, and $\Delta P_{G S V S C, i}$ are deviation in frequency, dc voltage, sum of generator power, sum of power demand, tie line power of the $I^{\text {th }} \mathrm{AC}$ area, and power deviation at $i^{\text {th }}$ GSVSC, respectively. $k_{I F, i}$ is the interaction factor, $T_{D C, p}$ is the DC grid time constant (typically in the range of $200-500 \mathrm{msec}$ for several hundred

TABLE I: SIMPLIFIED AC-MTDC GRID-1 PARAMETERS

\begin{tabular}{|l|c|c|l|c|}
\hline \multicolumn{3}{|c|}{ AC System } & \multicolumn{2}{c|}{ MTDC grids } \\
\hline Parameters & Area1 & Area2 & Parameters & Values \\
\hline$K_{p}(\mathrm{pu} / \mathrm{Hz})$ & 1 & 1 & $T_{D C}(\mathrm{sec})$ & 0.3 \\
\hline$T_{p}(\mathrm{sec})$ & 20 & 25 & $T_{G S V S C 1}(\mathrm{sec})$ & 6.8 \\
\hline$T_{g t 1,2}(\mathrm{sec})$ & 0.38 & - & $T_{G S V S C 2}(\mathrm{sec})$ & 6.475 \\
\hline$T_{g t 3,4}(\mathrm{sec})$ & - & $0.36,0.39$ & $T_{S C}(\mathrm{sec})$ & 0.2149 \\
\hline$T_{12}(\mathrm{pu} / \mathrm{Hz})$ & \multicolumn{2}{|c|}{0.245} & - & - \\
\hline $\mathrm{R}(\mathrm{Hz} / \mathrm{pu})$ & \multicolumn{2}{|c|}{2.4} & - & - \\
\hline
\end{tabular}




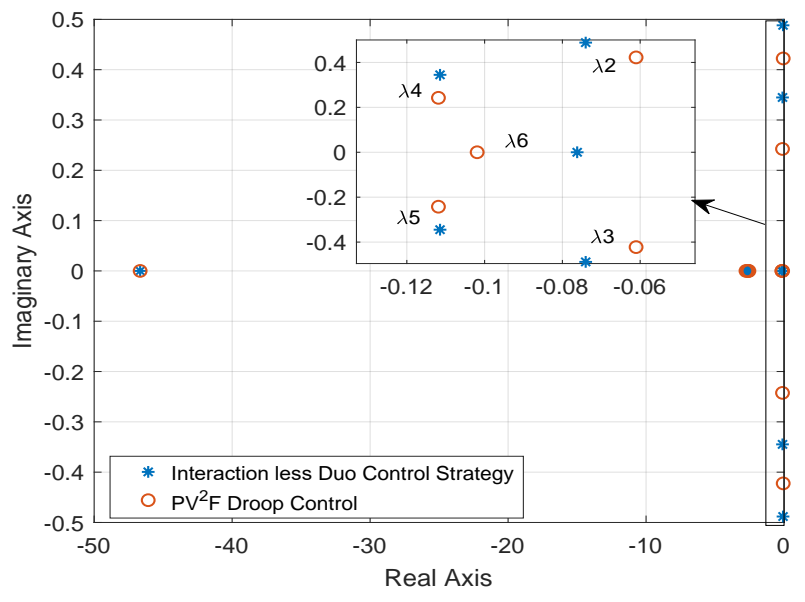

(a) Eigenvalue Trajectory

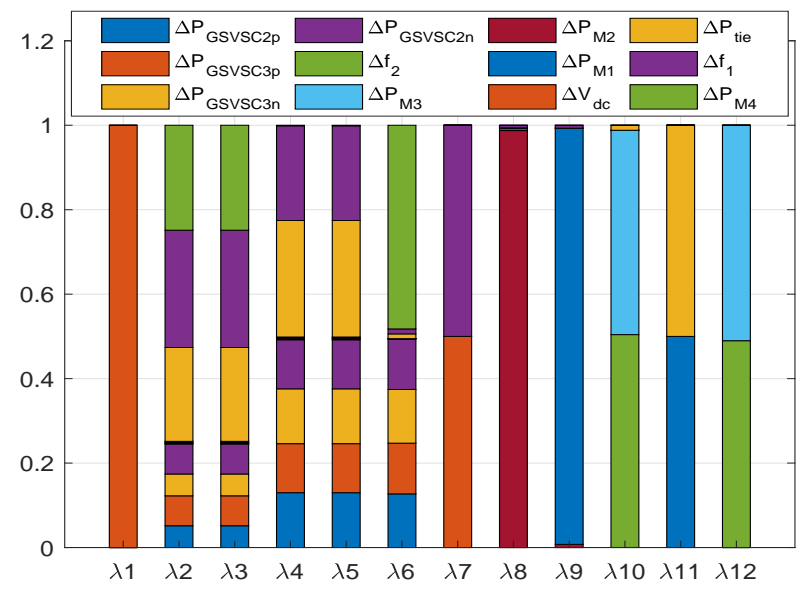

(b) Participation Factor

Fig. 3: Eigenvalue analysis of $P V^{2} F$ and proposed Duo control strategies for the test system AC-MTDC grid-1

megawatts power transfer [26]), $R_{I}$ is the $\mathrm{AC}$ frequency droop constants, $K_{p, I}$ is $\frac{1}{D_{I}}, T_{p, I}$ is $\frac{M_{I}}{D_{I}}=\frac{2 H_{I} / \omega_{0}}{D_{I}}$ (here $D_{I}$, and $M_{I}$ are the damping constant, and momentum of inertia) of the $I^{t h}$ area AC system. Moreover, $T_{g t, I}, T_{D C, i}$, and $T_{I J}$ are turbine governor, MTDC system time constant, and synchronizing power coefficient, respectively. The parameters of the simplified AC-MTDC grid-1 (in equations (15) - (22)) are given in TABLE I for both the $P V^{2} F$ and proposed Duo control strategies.

Further, the closed-loop state-space model can be written by using generalized equations (15)-(22), as

$$
\begin{gathered}
\dot{\mathbf{x}}=\mathbf{A x}+\mathbf{B u}, x\left(t_{0}\right)=0 \\
\mathbf{y}=\mathbf{C x} \\
\mathbf{P}=\mathbf{\Psi} \boldsymbol{\Phi}
\end{gathered}
$$

where, $\mathrm{x}$ is the state vector i.e., $\left[\begin{array}{lll}\Delta f_{1} & \Delta f_{2} & \Delta P_{G S V S C, p 1}\end{array}\right.$ $\Delta P_{G S V S C, n 1} \quad \Delta P_{G S V S C, p 2} \quad \Delta P_{G S V S C, n 2} \quad \Delta P_{m, 1} \quad \Delta P_{m, 2}$ $\left.\Delta P_{m, 3} \Delta P_{m, 4} \Delta P_{\text {tie }} \Delta V_{\mathrm{dc}}\right]^{\prime}$. The matrices $\mathbf{A}, \mathbf{B}$, and $\mathbf{C}$ are state, input, and output matrices, respectively. Whereas, $\mathbf{u}$ and $\mathbf{y}$ are input and output vectors. $\Psi$, and $\Phi$ are left, and right eigenvectors.

This state-space model and participation factor in (23) and (24) aids in analyzing the stability of the system and identifying sensitive state variables, respectively. The eigenvalue trajectory for the $P V^{2} F$ droop and the proposed Duo control methodolgies is as shown in Fig. 3(a). Whereas, the participation factor obtain is as in Fig. 3(b). From the eigenvalue trajectory and participation factor, it has been observed that the eigenvalues corresponding to the state-variables $\Delta \mathrm{f}_{1}, \boldsymbol{\Delta} \mathrm{f}_{2}, \Delta \mathrm{P}_{\mathrm{GSVSC}, 2 \mathrm{p}}, \boldsymbol{\Delta} \mathrm{P}_{\mathrm{GSVSC}, 2 \mathrm{n}}, \boldsymbol{\Delta} \mathrm{P}_{\mathrm{GSVSC}, 3 \mathrm{p}}$, $\Delta \mathbf{P}_{\text {GSVSC,3n }}$ and $\Delta \mathbf{P}_{\text {tie }}$ are more sensitive, and are associated with the eigen values $\lambda 2, \lambda 3, \lambda 4, \lambda 5$, and $\lambda 6$. Moreover, the dominant eigenvalues corresponding to interaction less Duo control strategy are towards the left-hand side in comparison with the $P V^{2} F$ droop control method. This plot also infers that the proposed Duo control strategy is more stable than the conventional droop control approach. It is achieved by nullifying the interaction in $P V^{2} F$ droop control by proposing a Duo control strategy for B-VSCs in the AC-MTDC grids.

\section{Simulation And Discussion}

The proposed Duo control strategy is validated using two test systems say AC-MTDC grid-1, and AC-MTDC grid-2, as shown in Fig. 1, and Fig. 4, respectively. In the AC-MTDC grid-1, the two area ac power system is considered, where bus 7 and 9 are connected to the GS-VSC3 and GS-VSC2 as in Fig. 1, and are operating at $220 \mathrm{kV}, 60 \mathrm{~Hz}$. The details of the two area power system are given in [27]. In case of the ACMTDC grid-2, the New England IEEE 39 bus is integrated into a five terminal CIGRE benchmark model. This ac and MTDC grid are interconnected through the GS-VSC2 and GS-VSC3, as shown in Fig. 4 and are operationg at $345 \mathrm{kV}, 60 \mathrm{~Hz}$. In both the test systems, the CIGRE B4 DC grid benchmark model is in common, which consists of a five terminal mesh type $\mathrm{Bi}$ pole DC grid operating at $\pm 400 \mathrm{kV}$. While the offshore ac grid is operating at $145 \mathrm{kV}$, and onshore ac grid connected to GS-VSC1 is operating at $380 \mathrm{kV}$. The control parameters details of B-VSC are tabulated in TABLE II.

To show outperform of the proposed Duo control strategy, two scenarios has been considered as in TABLE III. In scenario I (i.e., the proposed Duo control method) GS-VSC2 and 3 operates in the proposed control method. While in scenario II, the GS-VSC2 and GS-VSC3 are operates in the $P V^{2} F$ droop control method. In both the scenarios, the GSVSC1 operates in constant dc voltage control mode, WFVSC1, and WF-VSC2 operates in the constant power control mode. The set operating power of the GS-VSC2 and GSVSC3 is $300 \mathrm{MW}$, and WF-VSC1 and 2 are $400 \mathrm{MW}$, and $500 \mathrm{MW}$, respectively. Further, for the AC-MTDC grid-1, the droop constants $K_{V}$, and $K_{f}$ are chosen as 5 each for B-VSC

\section{TABLE II: CONTROL PARAMETERS OF VSC}

\begin{tabular}{lll}
\hline Symbol & Description & value \\
\hline & Outer Control loop parameters \\
\hline$K_{p}, T_{i}$ & Real and reactive power control & $0.001,0.0303$ \\
$K_{p}, T_{i}$ & AC voltage control & $1.0,0.1$ \\
$K_{p}, T_{i}$ & DC-voltage control & $14,0.2$ \\
\hline & Inner control loop parameters \\
\hline$K_{p d}, T_{i d}$ & Voltage d-axis control & $0.48,0.067$ \\
$K_{p d}, T_{i d}$ & Voltage q-axis control & $0.48,0.067$ \\
\hline
\end{tabular}




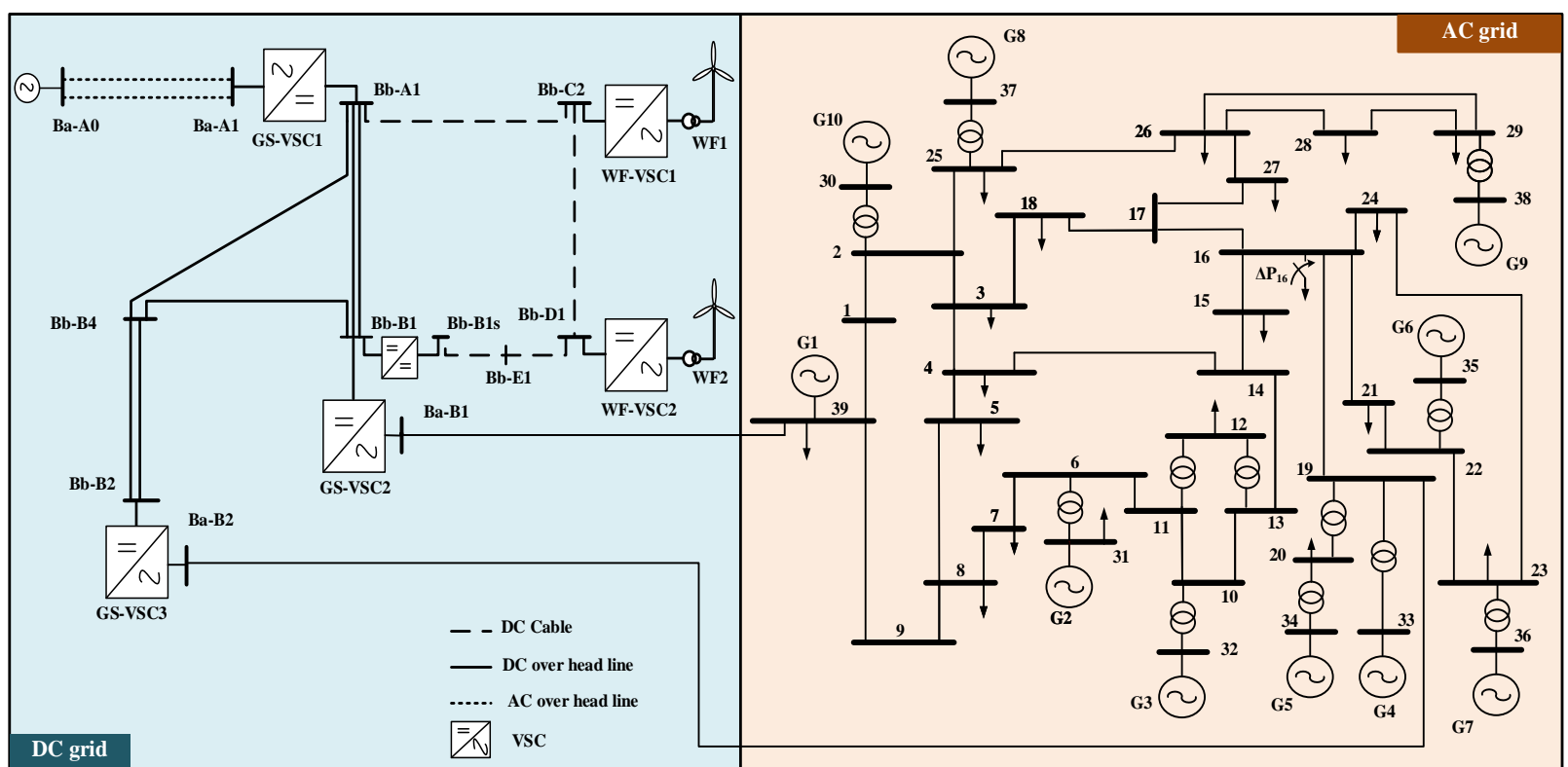

Fig. 4: AC-MTDC grid 2: Five terminal CIGRE B4 DC grid integrated into the New England IEEE 39 Bus System.

TABLE III: OPERATING MODES OF B-VSCs

\begin{tabular}{|c|c|c|}
\hline Converter & Scenario I & Scenario II \\
\hline GS-VSC1 & Vdc/Vac & Vdc/Vac \\
\hline GS-VSC2 & Duo & $\mathrm{PV}^{2} \mathrm{f}$ \\
\hline GS-VSC3 & Duo & $\mathrm{PV}^{2} \mathrm{f}$ \\
\hline WF-VSC1 & $P / V_{a c}$ & $P / V_{a c}$ \\
\hline WF-VSC2 & $P / V_{a c}$ & $P / V_{a c}$ \\
\hline
\end{tabular}

operating in the $P V^{2} F$ droop control. While in the proposed Duo control method to have a fair comparison, $K_{V p}$, and $K_{f n}$ are chosen as 10 each for positive, and negative pole operating in the $P V^{2}$, and PF droop control methods. Further, these two scenarios has been compared in three different case studies to show the efficacy of the proposed Duo control method.

\section{A. Case I: A step rise of $150 \mathrm{MW}$ power at bus 9 in the ac side of AC-MTDC grid 1}

Before step rise of a $150 \mathrm{MW}$, the AC-MTDC grid operating stable with ac load of $P_{7}+j Q_{7}=1267$ MW-j100 MVar and $P_{9}+j Q_{9}=1917 \mathrm{MW}-\mathrm{j} 250 \mathrm{MVar}$, and tie line power flow between bus 7 and 9 is $327 \mathrm{MW}-\mathrm{j} 48.62 \mathrm{MVar}$ in the two area power system. While GS-VSC2 and 3 are operating at $300 \mathrm{MW}$ each in inverter mode. In this scenario at $\mathrm{t}=$ $20 \mathrm{~s}$, a step rise of $\Delta P_{9}$ of $150 \mathrm{MW}$ has been considered. This cause the deficiency of a $150 \mathrm{MW}$ power in the MTDC grid. This deficiency of power reflects in the deviation of the frequency in the ac grid. However, the frequency regulation and support are enhanced with the proposed Duo control method in comparison with the as in $P V^{2} F$ droop control as in Fig. 5. The steady-state frequency before, and after the sudden step rise of load in scenario I (Proposed Duo control method) is $60.04 \mathrm{~Hz}$, and $59.83 \mathrm{~Hz}$ and scenario II $\left(P V^{2} F\right.$ droop control) is $60.02 \mathrm{~Hz}$ and $59.73 \mathrm{~Hz}$, respectively. Further, the frequency nadir are $59.75 \mathrm{~Hz}$, and $59.59 \mathrm{~Hz}$ in scenario I, and II, respectively.
As in Fig. 6, the sudden load rise also cause power deviation at the converter stations from its preset value in scenario I and II. The GS-VSC1 operates at $-79 \mathrm{MW}$, and $-85 \mathrm{MW}$ in scenario I, and II, respectively. While in scenario I, the GSVSC2 and 3 are operate at $-331 \mathrm{MW}$, and $-347 \mathrm{MW}$. Whereas in scenario II, the GS-VSC2 and GS-VSC3 are operate at $347 \mathrm{MW}$, and $-324 \mathrm{MW}$, respectively. This improved powersharing with the proposed Duo control strategy in comparison with $P V^{2} F$ droop control is responsible for maintaining the frequency in the AC-MTDC grid. In case of the dc voltage deviation, in scenario I and II are almost same and does not deviate much from its nominal values of $800 \mathrm{kV}$ as in Fig. 7. This is due to the presence of swing or slack converter (ie., GS-VSC1), and it is capable of regulating the dc voltage in the MTDC grid.

\section{B. Case II: A single pole outage of GS-VSC1 in the AC-MTDC grid 1}

This case is crucial to show the effectiveness of the proposed Duo control strategy. The GS-VSC1 is essential for regulating the $\mathrm{dc}$ voltage, and balancing the power-sharing in the $\mathrm{AC}$ MTDC grids. The outage of a single pole of GS-VSC1 causes surplus power in the MTDC grid, and it results in the deviation of frequency and dc voltage of the AC-MTDC grid. However, the improved frequency response is achieved with the proposed Duo control strategy as compared with the $P V^{2} F$ drop control method as shown in Fig. 8. The frequency zenith in scenario I and II after a single pole outage operating at $-90 \mathrm{MW}$ are $60.27 \mathrm{~Hz}$, and $60.54 \mathrm{H}$ respectively. Moreover, the steady-state frequency after an outage in scenario I and II are $60.19 \mathrm{~Hz}$, and $60.38 \mathrm{~Hz}$. From these, it is evident that the proposed Duo control strategy enhances the frequency support to the $\mathrm{AC}$ grid.

Before an outage of a VSC, the power-sharing by GS-VSC1, 2 , and 3 in scenario I, and II are $-180,-298$, and $-288 \mathrm{MW}$, respectively. After an outage of a pole in GS-VSC1, the power- 


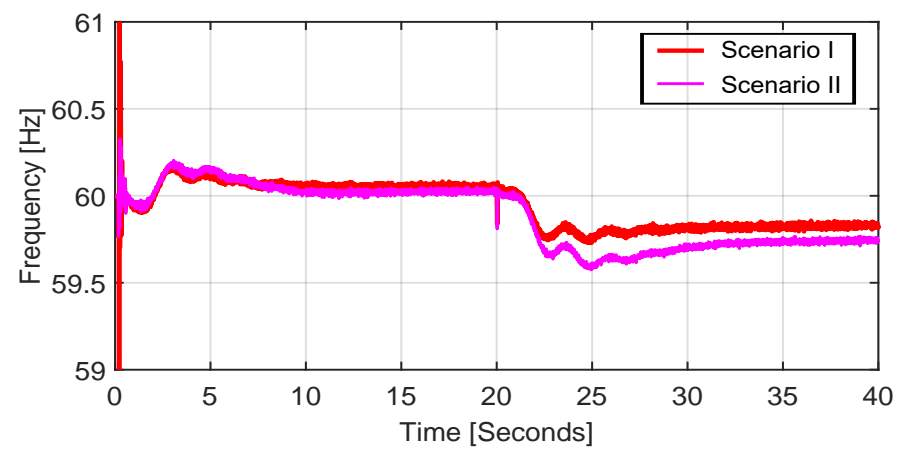

Fig. 5: Frequency support during two scenarios.

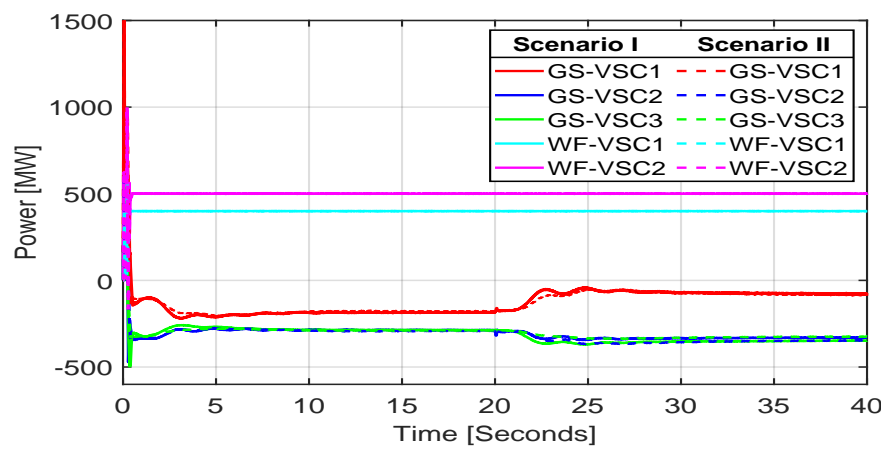

Fig. 6: Power flow between converter stations during two scenarios.

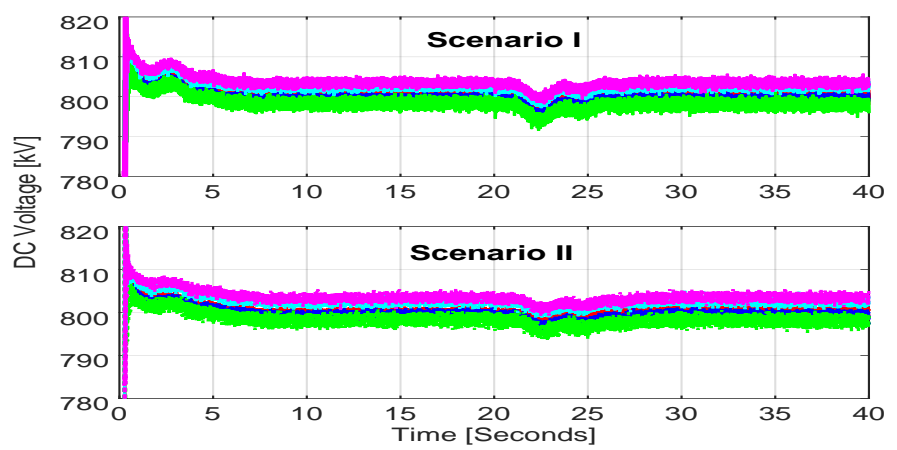

Fig. 7: DC-voltage at all converters stations during two scenarios.

sharing in scenario I by GS-VSC1, 2, and 3 are -146 MW, -371 MW, and -241 MW. While in scenario II, the power-shared by GS-VSC1, 2, and 3 are $-110 \mathrm{MW},-332 \mathrm{MW}$, and $-314 \mathrm{MW}$, respectively. The autonomous power-sharing is achieved with the proposed Duo control strategy (scenario I) in comparison with the $P V^{2} F$ droop control (scenario II) as in Fig. 9.

The improved dc voltage profile is also obtained by the proposed Duo control strategy in comparison with the $P V^{2} F$ droop control as shown in 10. After an outage of a pole, in scenario I and II, the dc voltage deviates to $808 \mathrm{kV}$, and 816 $\mathrm{kV}$ from its nominal value $800 \mathrm{kV}$, respectively.

It is evident from this case study that the proposed Duo control method not only improve the frequency but also improves the dc voltage profile along with autonomous powersharing in the AC-MTDC grid.

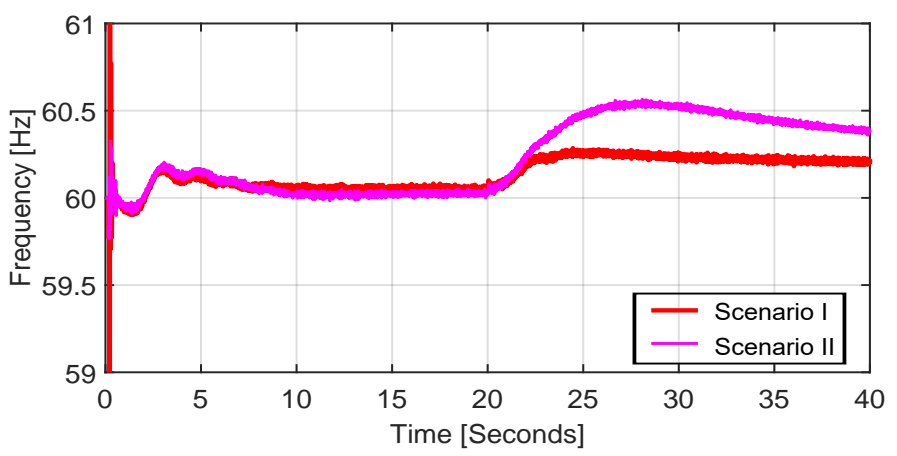

Fig. 8: Frequency support during two scenarios.

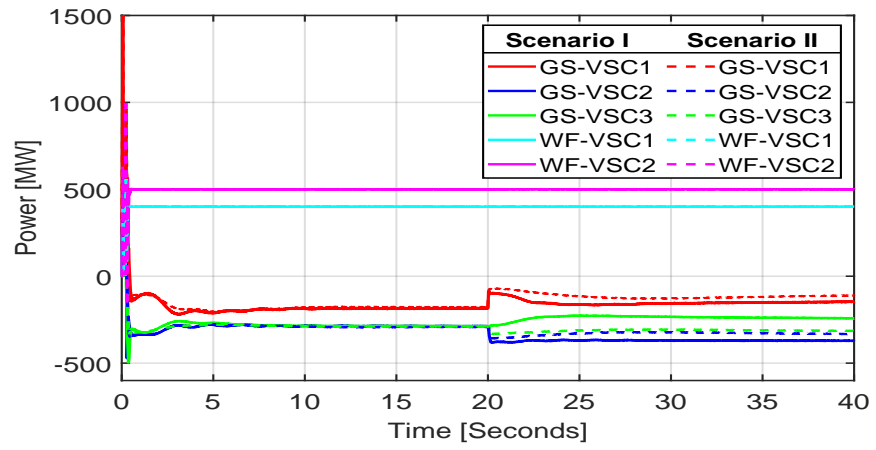

Fig. 9: Power flow between converter stations during two scenarios.

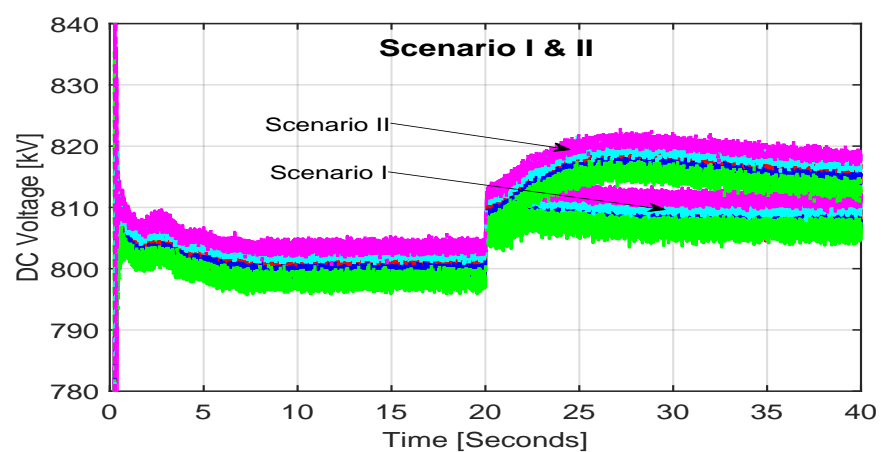

Fig. 10: DC-voltage at all converters stations during two scenarios.

C. Case III: A step rise of $600 \mathrm{MW}$ power at bus 16 in the ac side of AC-MTDC grid 2

The AC-MTDC grid-2 is considered to demonstrate the effectiveness of the proposed Duo control strategy in a proportional large ac system in comparison with the AC-MTDC grid 1. The GS-VSCs 2 and 3 are operating at set refernces of $500 \mathrm{MW}$ each, and are connected to the ac grid buses 39 and 19 , respectively. In this case, the droop constants $K_{V}$, and $K_{f}$ are chosen as 5 and 30 , respectively in scenario I. In the proposed Duo control method $K_{V p}$, and $K_{f n}$ are chosen as 10 , and 60 each for positive, and negative pole operating in the $P V^{2}$, and PF droop control methods. Further, the potency of the proposed method is shown while comparing with the $P V^{2} F$ droop control in scenario II.

In this case, a load switching of $600 \mathrm{MW}$ at bus 16 is examined in both scenarios. After step load change at $t=20$ $\mathrm{s}$, in scenario I, i.e., with the proposed Duo control strategy, 


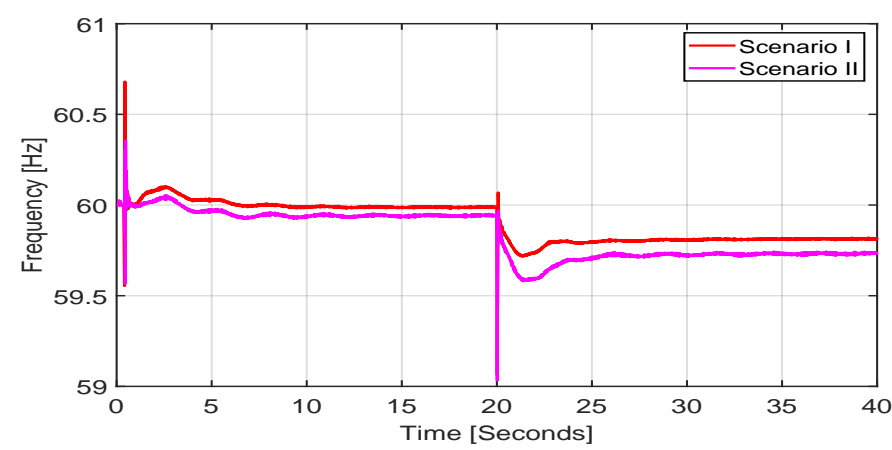

Fig. 11: Frequency support during two scenarios.

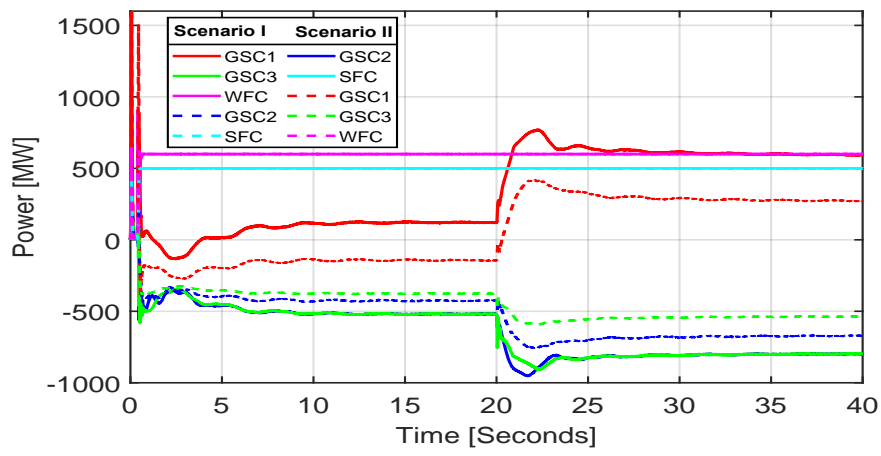

Fig. 12: Power flow between converter stations during two scenarios.

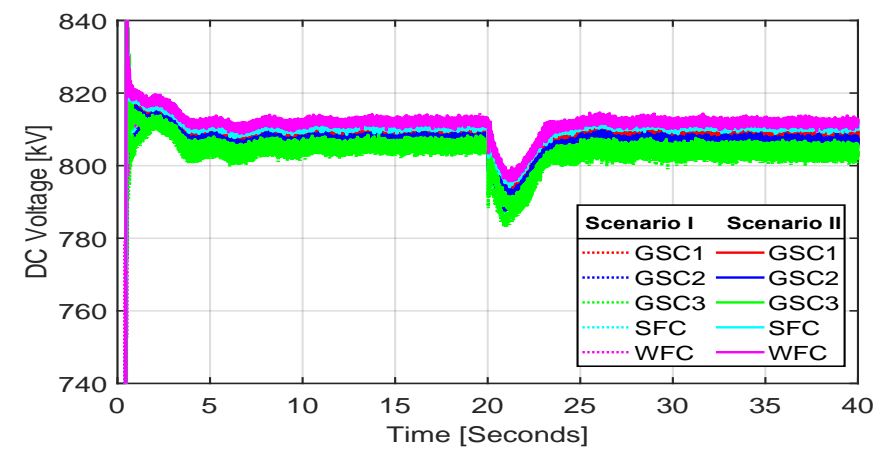

Fig. 13: DC-voltage at all converters stations during two scenarios.

an improved frequency profile is achieved compared with scenario II. As shown in Fig. 11, the frequency nadirs are 59.716 and $59.584 \mathrm{~Hz}$ in scenarios I and II. Besides, the steady-state frequencies are 59.80 and $59.73 \mathrm{~Hz}$, respectively. From these frequency attributes, one can infer that an improved frequency profile is achieved with the proposed Duo control strategy (scenario I) compared to the $P V^{2} F$ droop control (scenario II). This is realized through adequate power-sharing between terminals in the scenario I compared to scenario II, as in Fig. 12. The slack converter (GS-VSC1) regulates the dc voltage to its nominal value before and after the event as shown in Fig. 13.

Moreover, this case study infers that the proposed interaction less Duo control strategy performance surpasses the conventional $P V^{2} F$ droop control in the relatively large ac system integrated MTDC grid.

\section{CONCLusion}

The frequency and dc voltage support along with the autonomous power-sharing is very much essential for the stability, and reliability of the AC-MTDC grid. It has been accomplished by the proposed Duo control strategy while overcoming the drawbacks of conventional $P V^{2} F$ double droop control approach. To show the dominance of the proposed control, two scenarios, three case studies, and two differnt test systems has been considered. In this work, BVSC topology has been explored to achieve interaction less frequency and dc voltage support to the AC-MTDC grid. To show the dominance of the proposed control, two scenarios and various cases that include slack converter outage (GSVSC1) and switching of load have been considered for two test systems. The simulation results verify the analysis carried out in section III-C in support of the proposed Duo control strategy. The proposed control approach does not reliant on the dedicated communication links between converter stations, which reduces the installation cost of the system. Further, unlike in most of the literature, instead of regulating anyone control parameter among frequency and dc voltage, the proposed interaction less Duo control strategy is simple and yet capable of effectively regulating both the parameters frequency and $\mathrm{dc}$ voltage with autonomous power-sharing in the ACMTDC grids.

\section{REFERENCES}

[1] D. V. Hertem and M. Ghandhari, "Multi-terminal vsc hvdc for the european supergrid: Obstacles," Renewable and Sustainable Energy Reviews, vol. 14, no. 9, pp. 3156 - 3163, 2010.

[2] N. Chaudhuri, B. Chaudhuri, R. Majumder, and A. Yazdani, MultiTerminal Direct-Current Grids: Modeling, Analysis, and Control. United Kingdom: Wiley-Blackwell, 9 2014, vol. 9781118729106.

[3] T. E. COMMISSION, "Commission regulation (eu) 2016/1447 of 26 august 2016 establishing "establishing a network code on requirements for grid connection of high voltage direct current systems and direct current-connected power park modules"."

[4] N. R. Chaudhuri, R. Majumder, and B. Chaudhuri, "System frequency support through multi-terminal dc (mtdc) grids," IEEE Transactions on Power Systems, vol. 28, no. 1, pp. 347-356, Feb 2013.

[5] K. Rouzbehi, W. Zhang, J. Ignacio Candela, A. Luna, and P. Rodriguez, "Unified reference controller for flexible primary control and inertia sharing in multi-terminal voltage source converter-hvdc grids," IET Generation, Transmission Distribution, vol. 11, no. 3, pp. 750-758, 2017.

[6] O. D. Adeuyi, M. Cheah-Mane, J. Liang, and N. Jenkins, "Fast frequency response from offshore multiterminal vsc-hvdc schemes," IEEE Transactions on Power Delivery, vol. 32, no. 6, pp. 2442-2452, Dec 2017.

[7] K. Sun, K.-J. Li, W.-J. Lee, Z.-d. Wang, W. Bao, Z. Liu, and M. Wang, "Vsc-mtdc system integrating offshore wind farms based optimal distribution method for financial improvement on wind producers," IEEE Transactions on Industry Applications, vol. 55, no. 3, pp. 2232-2240, 2019.

[8] K. Rouzbehi, A. Miranian, J. I. Candela, A. Luna, and P. Rodriguez, "A generalized voltage droop strategy for control of multiterminal dc grids," IEEE Transactions on Industry Applications, vol. 51, no. 1, pp. 607-618, 2015.

[9] A. S. Kumar and B. P. Padhy, "Adaptive droop control strategy for autonomous power sharing and dc voltage control in wind farm-mtdc grids," IET Renewable Power Generation, vol. 13, no. 16, pp. 31803190, 2019.

[10] K. Shinoda, A. Benchaib, J. Dai, and X. Guillaud, "Over-and undervoltage containment reserves for droop-based primary voltage control of mtdc grids," IEEE Transactions on Power Delivery, 2021. 
[11] Z.-d. Wang, K.-J. Li, J.-g. Ren, L.-J. Sun, J.-G. Zhao, Y.-L. Liang, W.-J. Lee, Z.-h. Ding, and Y. Sun, "A coordination control strategy of voltage-source-converter-based mtdc for offshore wind farms," IEEE Transactions on Industry Applications, vol. 51, no. 4, pp. 2743-2752, 2015.

[12] W. Wang, Y. Li, Y. Cao, U. Häger, and C. Rehtanz, "Adaptive droop control of vsc-mtdc system for frequency support and power sharing," IEEE Transactions on Power Systems, vol. 33, no. 2, pp. 1264-1274, March 2018.

[13] P. McNamara and F. Milano, "Model predictive control-based agc for multi-terminal hvdc-connected ac grids," IEEE Transactions on Power Systems, vol. 33, no. 1, pp. 1036-1048, Jan 2018.

[14] A. Kirakosyan, E. F. El-Saadany, M. S. E. Moursi, and K. Al Hosani, "Dc voltage regulation and frequency support in pilot voltage droopcontrolled multiterminal hvdc systems," IEEE Transactions on Power Delivery, vol. 33, no. 3, pp. 1153-1164, June 2018.

[15] A. S. Kumar and B. P. Padhy, "Communication-free approach for frequency support in the mtdc grids - a comparative study," in 2020 21 st National Power Systems Conference (NPSC), 2020, pp. 1-6.

[16] Y. Phulpin, "Communication-free inertia and frequency control for wind generators connected by an hvdc-link," IEEE Transactions on Power Systems, vol. 27, no. 2, pp. 1136-1137, May 2012.

[17] B. Silva, C. L. Moreira, L. Seca, Y. Phulpin, and J. A. Pecas Lopes, "Provision of inertial and primary frequency control services using offshore multiterminal hvdc networks," IEEE Transactions on Sustainable Energy, vol. 3, no. 4, pp. 800-808, Oct 2012.

[18] P. Kou, D. Liang, Z. Wu, Q. Ze, and L. Gao, "Frequency support from a dc-grid offshore wind farm connected through an hvdc link: A communication-free approach," IEEE Transactions on Energy Conversion, vol. 33, no. 3, pp. 1297-1310, Sep. 2018.

[19] Y. Li, Z. Xu, J. Østergaard, and D. J. Hill, "Coordinated control strategies for offshore wind farm integration via vsc-hvdc for system frequency support," IEEE Transactions on Energy Conversion, vol. 32, no. 3, pp. 843-856, Sep. 2017.

[20] A. S. Kumar and B. P. Padhy, "Hybrid control strategy for effective frequency regulation and power sharing in multi-terminal hvdc grids," IET Generation, Transmission \& Distribution, vol. 14, no. 23, pp. 55365546, 2020.

[21] A. E. Leon, "Short-term frequency regulation and inertia emulation using an mmc-based mtdc system," IEEE Transactions on Power Systems, vol. 33, no. 3, pp. 2854-2863, May 2018.

[22] A. Bidadfar, O. Saborío-Romano, J. N. Sakamuri, N. A. Cutululis, V. Akhmatov, and P. E. Sørensen, "On feasibility of autonomous frequency-support provision from offshore hvdc grids," IEEE Transactions on Power Delivery, vol. 35, no. 6, pp. 2711-2721, 2020.

[23] A. S. Kumar and B. P. Padhy, "An interaction less duo control strategy for bi-polar voltage source converter in renewables integrated multiterminal hvdc (mtdc) grids," in 2020 IEEE International Conference on Power Electronics, Drives and Energy Systems (PEDES), 2020, pp. 1-5.

[24] H. Saad, J. Peralta, S. Dennetière, J. Mahseredjian, J. Jatskevich, J. A Martinez, A. Davoudi, M. Saeedifard, V. Sood, X. Wang, J. Cano, and A. Mehrizi-Sani, "Dynamic averaged and simplified models for mmc-based hudc transmission systems," IEEE Transactions on Power Delivery, vol. 28, no. 3, pp. 1723-1730, July 2013.

[25] J. Freytes, "Small-signal stability analysis of modular multilevel converters and application to mmc-based multi-terminal dc grids," Ph.D. dissertation, Ecole Centrale de Lille, 2017.

[26] E. Rakhshani, D. Remon, A. M. Cantarellas, J. M. Garcia, and P. Rodriguez, "Virtual synchronous power strategy for multiple hvdc interconnections of multi-area agc power systems," IEEE Transactions on Power Systems, vol. 32, no. 3, pp. 1665-1677, 2016.

[27] P. Kundur and N. Balu, Power System Stability and Control, ser. EPRI power system engineering series. McGraw-Hill, 1994. 